\title{
Analytical and Clinical Performance of the Nanopia Krebs von den Lungen 6 Assay in Korean Patients With Interstitial Lung Diseases
}

Eun-Jung Cho, M.D., Ph.D., ${ }^{1,2}$, Kyoung-Jin Park, M.D., Ph.D. ${ }^{3}$, Dae-Hyun Ko, M.D., Ph.D. ${ }^{3}$, Hyun Jung Koo, M.D., Ph.D. ${ }^{4}$, Sang Min Lee, M.D., Ph.D. ${ }^{4}$, Jin Woo Song, M.D., Ph.D. ${ }^{5}$, Woochang Lee, M.D., Ph.D. ${ }^{3}$, Hae Kyung Lee, M.D., Ph.D. ${ }^{1}$, Kyung-Hyun Do, M.D., Ph.D. ${ }^{4}$, Sail Chun, M.D., Ph.D. ${ }^{3}$, and Won-Ki Min $\mathbb{1}$, M.D., Ph.D. ${ }^{3}$

${ }^{1}$ Department of Laboratory Medicine, The Catholic University of Korea, Uijeongbu St. Mary's Hospital, Seoul, Korea; ${ }^{2}$ Department of Laboratory Medicine, Hallym University College of Medicine, Hwaseong, Korea; Departments of ${ }^{3}$ Laboratory Medicine, ${ }^{4}$ Radiology, and ${ }^{5}$ Pulmonary and Critical Care Medicine, Asan Medical Center, University of Ulsan College of Medicine, Seoul, Korea

Background: Krebs von den Lungen 6 (KL-6) is a sensitive marker for diagnosing, monitoring, and predicting the prognoses of interstitial lung diseases (ILDs). This study aimed to evaluate the performance of the Nanopia KL-6 assay (Sekisui Medical, Tokyo, Japan) and to test the relationship between $\mathrm{KL}-6$ concentrations and clinical results.

Methods: In total, 230 patients diagnosed as having ILDs were enrolled. All underwent high-resolution computed tomography (HRCT) followed by the pulmonary function test (PFT). We also enrolled 116 disease controls and 200 healthy controls. Evaluation of the Nanopia KL- 6 assay involved determination of precision, linearity, and limit of quantification (LOQ). Results from the Nanopia KL-6 assay were compared with those from ELISA and correlated with the HRCT and PFT results.

Results: The within-laboratory precisions were $<2 \%$ of $\mathrm{CV}$, and linearity was acceptable between 52.2 and $4,966.5 \mathrm{U} / \mathrm{mL}$. The LOQ was $45.2 \mathrm{U} / \mathrm{mL}$. Nanopia and ELISA results were strongly correlated $(r=0.979)$. The average concentration of $\mathrm{KL}-6$ was greater in ILD patients $(711.5 \mathrm{U} / \mathrm{mL})$ than in the disease $(168.4 \mathrm{U} / \mathrm{mL})$ and healthy $(209.4 \mathrm{U} / \mathrm{mL})$ controls. Serum KL-6 concentrations were strongly and moderately correlated with the extent of lung involvement and presence of typical HRCT abnormalities, respectively, and moderately correlated with PFT parameters.

Conclusions: The overall analytical and clinical performance of the Nanopia KL- 6 assay was acceptable. Our study is the first to compare assay platforms and show correlations between KL- 6 concentrations and HRCT or PFT results in Korean ILD patients.

Key Words: ELISA, High-resolution computed tomography, Interstitial lung diseases, Krebs von den Lungen 6 , Performance evaluation, Pulmonary function test

\author{
Received: February 5, 2018 \\ Revision received: June 26, 2018 \\ Accepted: December 13, 2018
}

Corresponding author: Won-Ki Min, M.D. (ib) https://orcid.org/0000-0002-5158-2130 Department of Laboratory Medicine, Asan Medical Center, University of Ulsan College of Medicine, 88 Olympic-ro 43-gil, Songpa-gu, Seoul 05505, Korea

Tel: +82-2-3010-4503

Fax: +82-2-478-0884

E-mail: wkmin@amc.seoul.kr

\section{INTRODUCTION}

Interstitial lung diseases (ILDs) comprise more than 200 diverse kinds of non-neoplastic or noninfectious diseases, which invade the lung interstitium [1]. These diseases can be broadly divided into four categories: ILDs with known causes, idiopathic intersti- tial pneumonia (IIP) of unknown cause, granulomatous ILD, and other forms of ILD [2]. ILDs are currently diagnosed using a multidisciplinary approach, which involves examination of clinical aspects, radiological and histological examinations, the pulmonary function test (PFT), and high-resolution computed tomography (HRCT) [3]. The HRCT features characteristic of ILD 
are reticular abnormality and honeycombing, often associated with traction bronchiectasis in patients with end-stage fibrotic lung disease. HRCT scans of the lung reveal patchy airspace consolidation, ground glass opacities in patients with early-stage ILD.

Krebs von den Lungen $6(\mathrm{KL}-6)$ is a high molecular weight glycoprotein found on the surface of diverse epithelial cell types. The surface expression of KL-6 is induced during the regeneration process of type II pneumocytes, resulting in an increased concentration in serum [1, 4]. In addition, the destruction of the air-blood barrier of affected lungs increases their permeability, leading to higher serum concentrations of KL-6 [4]. Thus, KL-6 has been reported as a useful biomarker for ILD diagnosis and for estimating disease severity and prognoses $[5,6]$. Detection of $\mathrm{KL}-6$, as a serological biomarker, is usually simple, noninvasive, and routinely available. Therefore, serum KL- 6 testing offers numerous advantages over traditional ILD assessment methods with better patient compliance. Several previous studies have reported a strong correlation between bronchoalveolar lavage fluid and serum concentrations of $\mathrm{KL}-6$, and it has been used in clinical research for diagnosing ILDs [7-9].

The National Health Insurance system of Japan has been using KL-6 as a diagnostic biomarker for ILD since 1999 [1]. Recently, with significant correlations being reported between higher KL-6 concentrations and reduction in PFT parameters, $\mathrm{KL}-6$ has been increasingly used for the prognosis and assessment of ILD severity $[8,10]$. Various commercial assays based on immunoassays, such as ELISA, electrochemiluminescence immunoassays, and latex particle-enhanced turbidimetric immunoassays (LTIAs), are currently used for measuring KL-6 concentrations [11, 12]. We evaluated the performance of the Nanopia KL-6 assay (Sekisui Medical, Tokyo, Japan), an LTIA, for measuring KL- 6 concentrations, and identified possible correlations between $\mathrm{KL}-6$ concentrations and clinical results obtained using HRCT and PFT. Clinical laboratories can use automated analyzers to perform the Nanopia KL-6 assay, which is faster and more convenient than ELISA. No study has compared LTIA and ELISA results for the measurement of KL- 6 concentrations. To our knowledge, this report is the first to compare the assay platforms, examining correlations between KL-6 concentrations and HRCT or PFT results in ILD patients.

\section{METHODS}

\section{Study population}

From June to December 2016, patients over 45 years of age who visited the pulmonology department of Asan Medical Center, Seoul, Korea were enrolled. We reviewed all patients' medical records and collected information including age, sex, results of HRCT and the PFT, and pathological results. Patients diagnosed as having ILDs and who underwent the PFT and/or had an HRCT examination were selected as the patient group. We included patients with diagnoses of ILDs confirmed clinically or histopathologically and excluded those with a previous diagnosis of cancer. In total, 230 patients underwent the PFT, and 201 were examined using HRCT. ILDs included six clinical-radiologic-pathologic entities: idiopathic pulmonary fibrosis (IPF), nonspecific interstitial pneumonia, cryptogenic organizing pneumonia, unclassifiable ILD, connective tissue disease (CTD)-associated ILD (CTD-ILD), and hypersensitivity pneumonitis (HP). Fewer than 10 patients had other interstitial pulmonary diseases and were assigned to the "others" category. CTDs included polymyositis/dermatomyositis, rheumatoid arthritis (RA), systemic lupus erythematosus, systemic sclerosis, Sjögren's syndrome, and undifferentiated CTD (UCTD). The patient group included 134 males and 96 females, and the median age was 64 years (range, 45-93 years). Age did not differ among the ILD subgroups $(P=0.236)$.

The control group was further divided into disease and healthy control groups. The disease control group comprised patients examined by X-ray or HRCT. Inclusion criteria for this group were the presence of infectious lung diseases, such as tuberculosis or pneumonia, chronic obstructive pulmonary disease (COPD), or radiation-induced pulmonary fibrosis (RIPF). Patients with a prior cancer diagnosis and cardiovascular disease were excluded from the study; however, an exception was made for the RIPF group. The healthy control group comprised adult patients $>45$ years of age who visited the health screening and promotion center of Asan Medical Center during the same period for regular health examinations. Exclusion criteria for the healthy control group comprised a concomitant chronic condition such as current or previous record of hypertension, diabetes mellitus, renal disease, or malignant tumors and abnormal chest X-ray or chest CT results.

The clinical protocol and design of this study were approved by the Institutional Review Board of Asan Medical Center (approval number: 2016-0377). All patients, disease controls, and healthy controls gave written informed consent.

\section{Laboratory measurements}

We collected residual samples after chemical analysis. We collected 230 samples for ILD patients and 116 and 200 samples 
for disease and healthy controls, respectively. Serum was aliquoted and stored at $-70^{\circ} \mathrm{C}$. Serum KL-6 concentration was measured using the AU5822 analyzer (Beckman Coulter, Brea, CA, USA) with the Nanopia KL-6 assay. KL- 6 in the samples agglutinates with the mouse KL-6 monoclonal antibody-coated latex through the antigen-antibody reaction. The change in absorbance caused by this agglutination is measured to determine the $\mathrm{KL}-6$ concentration. For comparison, the $\mathrm{KL}-6$ concentration was also measured using a manual KL-6 ELISA kit (EIDIA, Tokyo, Japan). KL-6 assays were performed as per the manufacturers' instructions. The cut-off value for both the Nanopia and ELISA assays, as recommended by the manufacturers, was 500 $\mathrm{U} / \mathrm{mL}$.

\section{Precision}

Precision was evaluated according to the CLSI EP5-A3 guidelines [13]. Quality control (QC) materials corresponded to two concentrations of the assay. The test was performed with a target mean of 461 (range 369-553 U/mL) for Level 1 QC material and 1,089 (range 871-1,307 $\mathrm{U} / \mathrm{mL}$ ) for Level $2 \mathrm{QC}$ material, which were included in the Nanopia KL-6 assay kit. Two replicates of each control sample were analyzed twice per day for 20 days, with the two runs separated by at least two hours.

\section{Linearity}

Linearity was evaluated according to the CLSI EP6-A guideline [14]. We used five concentrations of the Nanopia KL-6 calibrators (Sekisui Medical). Two repeat measurements of each concentration were analyzed.

\section{Limit of quantification}

Limit of quantification (LOQ), defined as the minimum concentration with a CV $<20 \%$, was determined by repeated measurements for eight days with one run. Target concentrations were approximately 44.0, 48.0, 50.0, 52.0, and 58.0 U/mL. For each concentration, pooled serum samples were aliquoted into eight samples.

\section{Comparison studies}

Assay methods were compared according to the CLSI EP9-A3 guidelines [15]. We collected 50 patient samples in the clinically relevant concentration ranges (i.e., reference range) and analyzed correlations between the results obtained with the Nanopia KL-6 assay and ELISA.

\section{HRCT}

The extent of HRCT abnormalities was determined by visually estimating the percentage of lung involvement using a semiquantitative scoring system. HRCT results were graded on a scale of 1-6 as follows: 1) normal attenuation, 2) ground-glass attenuation, 3) consolidation, 4) reticular abnormality, 5) traction bronchiectasis, and 6) honeycombing [16]. The fibrotic score (\%), which was the sum of the honeycombing and reticulation scores, was also calculated [17].

\section{PFT}

Spirometry, total lung capacity (TLC) by plethysmography and diffusing capacity of the lung for carbon monoxide ( $D L_{c o}$ ) were measured, and the results were expressed as percentages of normal predicted values [18]. The 6-minute walking distance (6MWD) was determined according to the ATS guidelines with a minor modification [19]. Briefly, the technician followed the patients with continuous monitoring of oxygen saturation but offered no additional remark or encouragement.

\section{Statistical analysis}

To compare KL-6 concentrations among patients, disease controls, and healthy controls, a one-way ANOVA was performed, followed by the Bonferroni post hoc test. The normality of the data was assessed by the Shapiro-Wilk. Spearman correlation analyses were performed between KL-6 concentrations and HRCT and PFT results. EP Evaluator Release 8 (David G. Rhoads Associates, Kennett Square, PA, USA), SPSS for Windows (ver. 18.0; SPSS Inc., Chicago, IL, USA), and Microsoft Excel 2013 (Microsoft Corp., Redmond, WA, USA) were used for the statistical analyses. $P<0.05$ was considered statistically significant. To compare the Nanopia KL-6 assay and ELISA, we performed Deming regression using EP Evaluator Release 8. This program detected outliers in a method comparison experiment and excluded them from the data set. Bland-Altman plots were constructed to interpret the differences in results across assays.

\section{RESULTS}

\section{Analytical performance of the Nanopia KL-6 assay}

The means $( \pm \mathrm{SD})$ of the two QC materials were $485.1( \pm 8.6)$ $\mathrm{U} / \mathrm{mL}$ and $1,102.7( \pm 17.6) \mathrm{U} / \mathrm{mL}$. The $\mathrm{CV} \%$ of reproducibility and within-laboratory precision were $1.1 \%$ and $1.8 \%$ at low concentrations and $0.9 \%$ and $1.6 \%$ at high concentrations, respectively. The linear range where the coefficient of determina- 
A

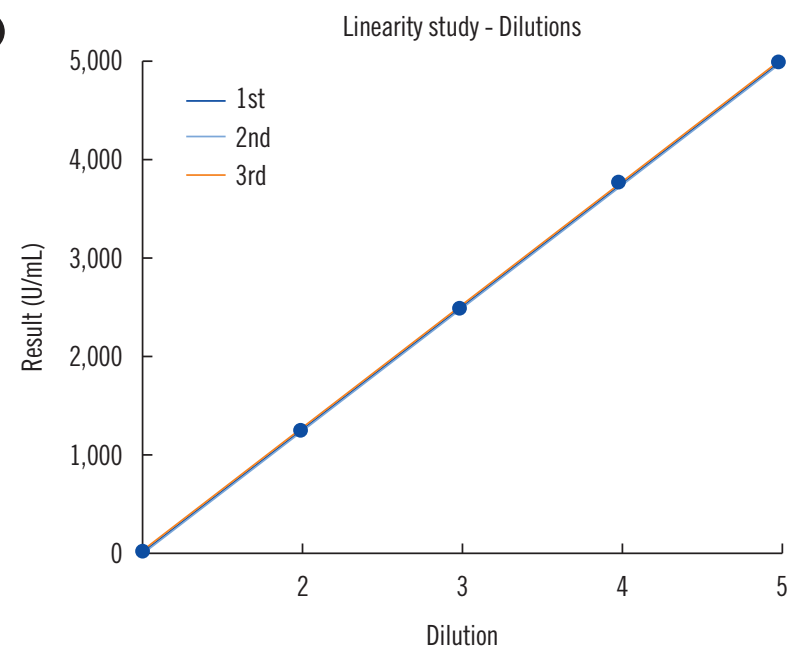

B

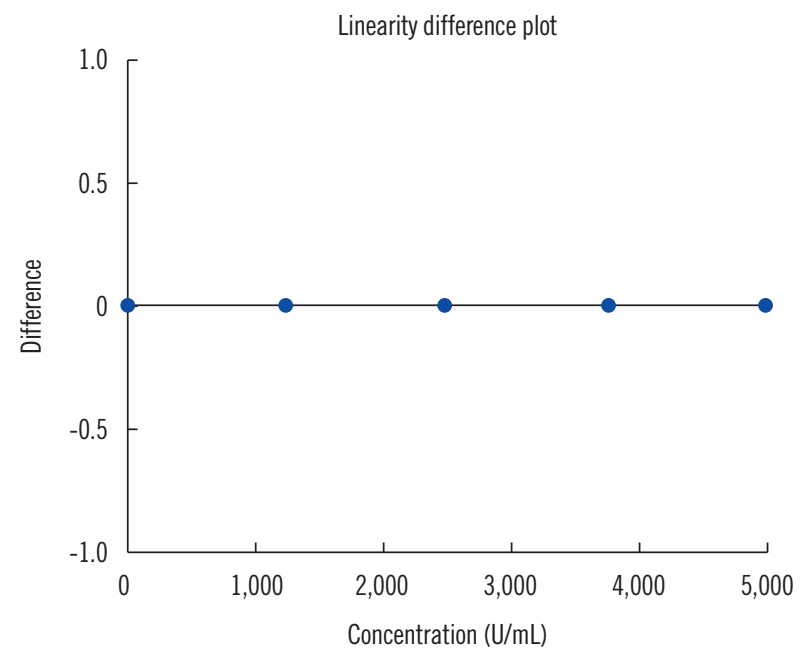

Fig. 1. Linearity of the Nanopia Krebs von den Lungen 6 (KL-6) assay as estimated using the AU5822 analyzer (Beckman Coulter, Brea, CA, USA): the third-, second- and first-order models (A) and a difference plot (B) between the first- and third-order models at each concentration obtained from polynomial regression analysis.

A

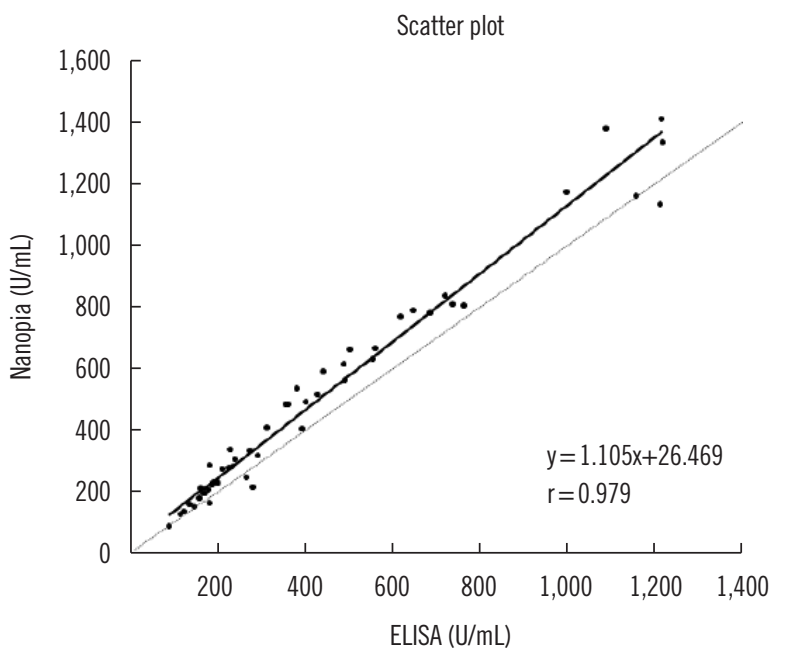

B

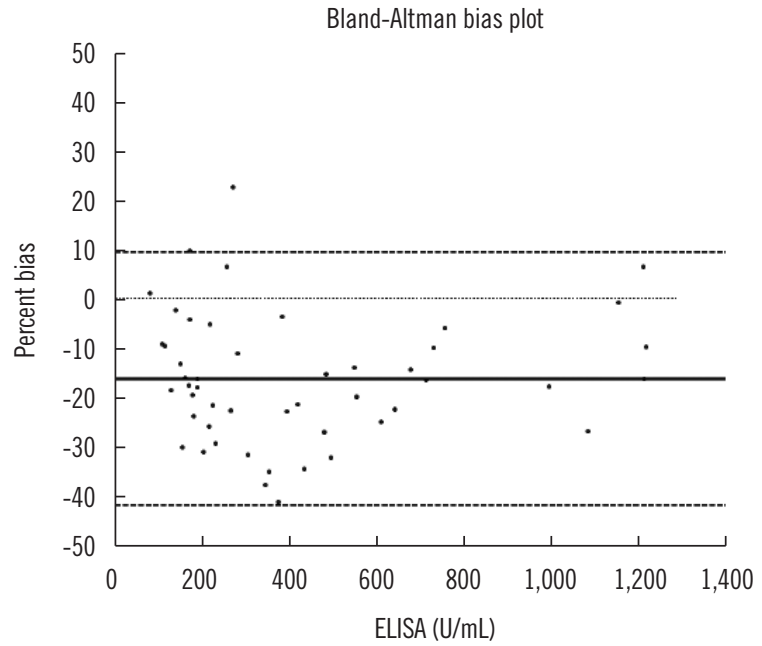

Fig. 2. Scatter plots (A) and Bland-Altman bias plots (B) of the Krebs von den Lungen 6 (KL-6) concentrations of the automated Nanopia $\mathrm{KL}-6$ assay and the manual $\mathrm{KL}-6$ ELISA $(\mathrm{N}=50)$. In the scatter plot, the solid line is the Deming regression line, and dotted line is the $1: 1$ line. In the Bland-Altman plot, the solid line represents the average percent bias, dotted line is the zero line, and dashed lines are upper and lower 95\% confidence limits for the mean difference (\%). Percent bias was calculated using the following formula: (ELISA-Nanopia)/ ELISA $\times 100(\%)$.

tion $\left(R^{2}\right)$ is slightly greater than 0.99 was $52.2-4,966.5 \mathrm{U} / \mathrm{mL}$. The best-fitted curve was linear (Fig. 1). The LOQ was $45.2 \mathrm{U} /$ $\mathrm{mL}$. The correlation coefficient $(r)$ between the Nanopia and ELISA results was 0.979 (Fig. 2). The Nanopia assay showed a higher KL-6 concentration than the ELISA kit.

Differences in serum KL-6 concentrations between patients, disease controls, and healthy controls

The mean KL- 6 concentrations of the ILD patients and the disease and healthy control groups were 711.5, 209.4, and 168.4
$\mathrm{U} / \mathrm{mL}$, respectively (Table 1 ). $\mathrm{KL}-6$ concentrations of the ILD patient subgroups are summarized in Table 1 . The data followed a normal distribution.KL-6 concentrations differed among ILD subgroups $(P=0.050)$. KL-6 concentrations of the IPF and HP groups were higher than those of the unclassifiable patients with $\operatorname{ILD}(P=0.03$ and $P=0.005$, respectively). Although the $\mathrm{KL}-6$ concentrations of the patients with IPF were greater than those of the other ILD subgroups, the differences were not significant. The difference in KL-6 concentrations among the type of CTDs in CTD-ILD did not reach statistical significance because of the 
Table 1. KL-6 concentrations in interstitial lung disease patients, disease controls, and healthy controls

\begin{tabular}{|c|c|c|c|c|c|c|}
\hline Group & & $\mathrm{N}$ & Mean & SD & $P$ & $P$ (vs control group) \\
\hline \multirow[t]{7}{*}{ ILD patients } & IPF & 52 & 912.2 & 557.8 & 0.050 & $<0.001$ (vs disease control group) \\
\hline & NSIP & 14 & 818.0 & 620.5 & & $<0.001$ (vs healthy control group) \\
\hline & COP & 19 & 670.5 & 527.3 & & \\
\hline & Unclassifiable & 68 & 514.8 & 494.8 & & \\
\hline & CTD-ILD & 44 & 685.3 & 566.3 & & \\
\hline & $\mathrm{HP}$ & 10 & 749.3 & 275.8 & & \\
\hline & Others & 23 & 743.0 & $1,108.2$ & & \\
\hline \multirow[t]{3}{*}{ Disease control group } & Infectious diseases & 60 & 188.6 & 132.1 & 0.215 & 0.027 (vs healthy control group) \\
\hline & Airway disease & 27 & 249.0 & 140.7 & & \\
\hline & RIPF & 29 & 215.4 & 159.8 & & \\
\hline Healthy control group & Healthy control & 200 & 168.4 & 62.4 & - & - \\
\hline
\end{tabular}

Abbreviations: KL-6, Krebs von den Lungen 6; ILD, interstitial lung disease; RIPF, radiation-induced pulmonary fibrosis; COP, cryptogenic organizing pneumonia; CTD-ILD, connective tissue disease-associated interstitial lung disease; HP, hypersensitivity pneumonitis; ILD, interstitial lung disease; IPF, idiopathic pulmonary fibrosis; NSIP, nonspecific interstitial pneumonia; Unclassifiable, unclassifiable interstitial lung diseases.

Table 2. Spearman's rank correlation analyses between serum KL-6 concentration and HRCT results

\begin{tabular}{lc}
\hline HRCT results & Correlation coefficient $(P)$ \\
\hline Percentage of lung involvement & $0.561(<0.001)$ \\
Ground-glass attenuation & $0.373(<0.001)$ \\
Reticular abnormality & $0.362(<0.001)$ \\
Traction bronchiectasis & $0.462(<0.001)$ \\
Consolidation & $0.048(0.496)$ \\
Honeycombing & $0.222(0.002)$ \\
Fibrotic score & $0.349(<0.001)$ \\
\hline
\end{tabular}

Abbreviations: KL-6, Krebs von den Lungen 6; HRCT, high-resolution computed tomography.

small sample size; however, KL-6 concentrations of patients with RA were lower than those of patients with UCTD.

Correlations between $\mathrm{KL}-6$ concentrations and HRCT results The KL-6 concentration showed a strong correlation with extent of lung lesions on HRCT (Table 2). It was not strongly correlated with the extent of airspace consolidation but was strongly correlated with other abnormal HRCT results. The fibrotic score (\%) also had a weak correlation with KL-6 concentration.

\section{Correlations between $\mathrm{KL}-6$ concentrations and PFT results}

Table 3 shows correlations between KL- 6 concentration and PFT results for the ILD subgroups that contained more than 10 patients each. The KL-6 concentration had a moderate correlation with the forced vital capacity (FVC), DLco, TLC, 6MWD, and oxygen blood saturation $\left(\mathrm{SpO}_{2}\right)$ in patients with IPF, CTD-ILD,
Table 3. Spearman's rank correlation analyses between serum KL-6 concentration and pulmonary function parameters

\begin{tabular}{|c|c|c|c|c|c|}
\hline \multirow{2}{*}{$\begin{array}{l}\text { Disease } \\
\text { category (N) }\end{array}$} & \multicolumn{5}{|c|}{ Correlation coefficient $(P)$} \\
\hline & FVC (\%) & $\mathrm{DL}_{\mathrm{co}}(\%)$ & TLC (\%) & 6MWD & $\begin{array}{c}\text { Minimum } \\
\mathrm{SpO}_{2}\end{array}$ \\
\hline \multirow[t]{2}{*}{ ILD (230) } & -0.493 & -0.523 & -0.418 & -0.320 & -0.557 \\
\hline & $(<0.001)$ & $(<0.001)$ & $(<0.001)$ & $(<0.001)$ & $(<0.001)$ \\
\hline \multirow{2}{*}{$\begin{array}{l}\text { Unclassifiable } \\
\text { (68) }\end{array}$} & -0.392 & -0.470 & -0.403 & -0.181 & -0.495 \\
\hline & -0.001 & $(<0.001)$ & -0.001 & -0.091 & $(<0.001)$ \\
\hline \multirow[t]{2}{*}{ IPF (52) } & -0.359 & -0.453 & -0.403 & -0.463 & -0.519 \\
\hline & -0.006 & -0.001 & -0.002 & $(<0.001)$ & $(<0.001)$ \\
\hline \multirow[t]{2}{*}{ CTD-ILD (44) } & -0.641 & -0.640 & -0.273 & -0.383 & -0.525 \\
\hline & $(<0.001)$ & $(<0.001)$ & -0.038 & -0.006 & $(<0.001)$ \\
\hline \multirow[t]{2}{*}{ COP (19) } & -0.591 & -0.411 & -0.203 & 0.281 & 0.040 \\
\hline & -0.004 & -0.040 & -0.203 & -0.122 & -0.435 \\
\hline \multirow[t]{2}{*}{ NSIP (14) } & -0.565 & -0.634 & -0.612 & -0.481 & -0.599 \\
\hline & -0.018 & -0.007 & -0.01 & -0.041 & -0.012 \\
\hline \multirow[t]{2}{*}{$\mathrm{HP}(10)$} & -0.081 & -0.286 & -0.713 & -0.399 & -0.408 \\
\hline & -0.412 & -0.211 & -0.010 & -0.126 & -0.121 \\
\hline
\end{tabular}

Abbreviations: KL-6, Krebs von den Lungen 6; COP, cryptogenic organizing pneumonia; CTD-ILD, connective tissue disease-associated interstitial lung disease; $\mathrm{DL}$ co $(\%)$, percentage predicted diffusing capacity of the lung for carbon monoxide; FVC (\%), percentage predicted forced vital capacity; HP, hypersensitivity pneumonitis; IPF, idiopathic pulmonary fibrosis; 6MWD, 6 minutes walking distance; NSIP, nonspecific interstitial pneumonia; $\mathrm{SpO}_{2}$, oxygen blood saturation; TLC (\%), percentage predicted total lung capacity; Unclassifiable, unclassifiable interstitial lung diseases.

and nonspecific interstitial pneumonia. The KL-6 concentration negatively correlated with FVC and DLco in cryptogenic organiz- 
ing pneumonia patients and with TLC in HP patients. In unclassifiable ILD patients, $\mathrm{FVC}, \mathrm{DL}_{\mathrm{co}}$, and $\mathrm{SpO}_{2}$ were negatively correlated with $\mathrm{KL}-6$ concentration (all $P<0.05$ ).

\section{DISCUSSION}

The average KL- 6 concentration in ILD patients was greater than that in the healthy and disease control groups, and in RIPF patients who received radiation treatment for lung or breast cancer. Though the majority of the ILD patients ( $\mathrm{N}=106 ; 46.1 \%$ ) were treated with medications, and the patients responding well to treatment showed decreased KL-6 concentrations [20], KL-6 concentrations significantly differed among groups. Moreover, while a cut-off value of $500 \mathrm{U} / \mathrm{mL}$ was recommended by the manufacturer, only one patient from the healthy control group and seven patients from the disease control groups had KL-6 concentrations above the cut-off. Increased KL- 6 concentrations have been observed in patients with advanced non-small cell lung cancer [20] and in patients with radiation pneumonitis, which is an acute manifestation of radiation-induced lung disease that can begin after radiation treatment [21]. Thus, ILDs may be difficult to be distinguished from such diseases based on serum KL-6 concentrations alone. In the present study, RIPF patients with late radiationinduced lung injury showed lower KL-6 concentrations than ILD patients $(P<0.01)$. Despite the limitations of this study, which included a small group of RIPF patients, the KL- 6 concentration served as a useful marker for differential diagnosis of ILD from RIPF in patients who suffered from chronic fibrosis in the lung.

There are no guidelines for evaluating KL- 6 assays. The total CVs for Nanopia KL- 6 assay and ELISA kit precision are $\leq 2 \%$ and $\leq 10 \%$, respectively, according to the manufacturers' instructions; therefore, a total $\mathrm{CV}$ of $<2 \%$ can be regarded as excellent for KL-6 concentrations, considering the analytes secreted from tissues [22].

The linearity of KL- 6 values was excellent, with a slope of 0.987 and an intercept of -0.1 , both of which were in the clinically relevant range. Moreover, Nanopia and ELISA results were strongly correlated $(r>0.975)$. However, the KL-6 concentrations determined by the Nanopia KL- 6 assay were higher than those by the ELISA kit. The difference may be related to the occurrence of a nonspecific reaction due to interference from the heterophile antibodies or rheumatoid factor. The removal of the Fc fragment from the antibody bound to the latex in the LTIA method may reduce interference [23]. Therefore, LTIA would provide more accurate results than ELISA; however, both methods use the same antibodies.
Our results were consistent with the previous results that serum concentrations of KL- 6 correlated with overall degree of lung involvement and presence of typical HRCT abnormalities $[20,24]$. A previous study found a positive correlation between the $\mathrm{KL}-6$ concentration and the extent of ground-glass opacity and traction bronchiectasis $(r=0.574$ and $r=0.617$, respectively; both $P<0.05$ ) in 26 patients with fibrotic nonspecific interstitial pneumonia [20]. In another study, scores for the extent of reticular patterning and honeycombing were associated with higher KL-6 concentrations in IPF ( $r=0.62$ and $r=0.41$, respectively; both $P<0.05$ ) [25]. In another study on patients with systemic sclerosis associated with ILD, the $\mathrm{KL}-6$ concentration was correlated with fibrosis score on HRCT $(r=0.68 ; P<0.05)$ [26].

Several studies have also reported a correlation between the restrictive pattern abnormalities on PFT and KL- 6 concentrations. Bonella et al. [26] reported that reduced FVC and DLco were correlated with increased KL-6 concentrations in systemic sclerosis patients with ILD ( $r=-0.47$ and $r=-0.58$, respectively; both $P<0.05$ ). In addition, Kuwana et al. [27] reported that the serum KL-6 concentration at diagnosis could serve as a useful biomarker for predicting progression to end-stage lung disease due to a subsequent reduction in the FVC $(r=0.58, P<0.05)$.

In 2014, two antifibrotic agents, pirfenidone and nintedanib, were approved by the US Food and Drug Administration for the treatment of IPF; thus, monitoring responses to these treatments becomes increasingly important [28]. Decreased KL-6 concentrations have been associated with survival benefits; thus, continuous monitoring after starting therapy is required [29].

In conclusion, the Nanopia KL-6 assay showed good precision, linearity (covering all measurement concentrations), and correlations with ELISA. These analytical and clinical characteristics may render this assay suitable for assessing ILD severity.

\section{Authors' Disclosures of Potential Conflicts of Interest}

The authors declare that there are no conflicts of interest.

\section{Acknowledgements}

The authors would like to acknowledge Kyung II Medical Inc. and Sekisui Medical Co., Ltd., for technical help.

\section{REFERENCES}

1. Kohno N, Awaya Y, Oyama T, Yamakido M, Akiyama M, Inoue Y, et al. 
KL-6, a mucin-like glycoprotein, in bronchoalveolar lavage fluid from patients with interstitial lung disease. Am Rev Respir Dis 1993;148:63742.

2. American Thoracic Society, European Respiratory Society International Multidisciplinary Consensus Classification of the Idiopathic Interstitial Pneumonias. This joint statement of the American Thoracic Society (ATS), and the European Respiratory Society (ERS) was adopted by the ATS board of directors, June 2001 and by the ERS Executive Committee, June 2001. Am J Respir Crit Care Med 2002;165:277-304.

3. National Clinical Guideline C. National institute for health and care excellence: Clinical guidelines. In: Diagnosis and management of suspected idiopathic pulmonary fibrosis: Idiopathic pulmonary fibrosis. London: Royal College of Physicians (UK) National Clinical Guideline Centre, 2013;44-51.

4. Meyer KC. Diagnosis and management of interstitial lung disease. Transl Respir Med 2014;2:4.

5. Huang $\mathrm{H}$, Peng X, Nakajima J. Advances in the study of biomarkers of idiopathic pulmonary fibrosis in Japan. Biosci Trends 2013;7:172-7.

6. Ishikawa N, Hattori N, Yokoyama A, Kohno N. Utility of KL-6/MUC1 in the clinical management of interstitial lung diseases. Respir Investig 2012;50:3-13.

7. Ichiyasu H, Ichikado K, Yamashita A, Iyonaga K, Sakamoto O, Suga M, et al. Pneumocyte biomarkers KL- 6 and surfactant protein $D$ reflect the distinct findings of high-resolution computed tomography in nonspecific interstitial pneumonia. Respiration 2012;83:190-7.

8. Wakamatsu K, Nagata N, Kumazoe H, Oda K, Ishimoto H, Yoshimi M, et al. Prognostic value of serial serum KL- 6 measurements in patients with idiopathic pulmonary fibrosis. Respir Investig 2017;55:16-23.

9. Ishii H, Mukae H, Kadota J, Kaida H, Nagata T, Abe K, et al. High serum concentrations of surfactant protein $\mathrm{A}$ in usual interstitial pneumonia compared with non-specific interstitial pneumonia. Thorax 2003;58: $52-7$.

10. du Bois RM, Weycker D, Albera C, Bradford WZ, Costabel U, Kartashov $A$, et al. Forced vital capacity in patients with idiopathic pulmonary fibrosis: test properties and minimal clinically important difference. Am J Respir Crit Care Med 2011;184:1382-9.

11. Hamai K, Iwamoto H, Ishikawa N, Horimasu Y, Masuda T, Miyamoto S, et al. Comparative study of circulating MMP-7, CCL18, KL-6, SP-A, and SP-D as disease markers of idiopathic pulmonary fibrosis. Dis Markers 2016;2016:4759040.

12. Chiba S, Ohta H, Abe K, Hisata S, Ohkouchi S, Hoshikawa Y, et al. The diagnostic value of the interstitial biomarkers KL- 6 and SP-D for the degree of fibrosis in combined pulmonary fibrosis and emphysema. Pulm Med 2012;2012:492960.

13. CLSI. Evaluation of precision performance of quantitative measurement methods; Approved guideline. 2nd ed. CLSI document EP5-A3. Wayne, PA: Clinical and Laboratory Standards Institute. 2014

14. CLSI. Evaluation of the linearity of quantitative measurement procedures: a statistical approach; Approved guideline. CLSI document EP6-A. Wayne,
PA: Clinical and Laboratory Standards Institute. 2003.

15. CLSI. Measurement procedure comparison and bias estimation using patient samples; Approved guideline. 3rd ed. CLSI document EP09-A3. Wayne, PA: Clinical and Laboratory Standards Institute. 2013.

16. Ichikado K, Johkoh T, Ikezoe J, Takeuchi N, Kohno N, Arisawa J, et al. Acute interstitial pneumonia: high-resolution CT findings correlated with pathology. AJR Am J Roentgenol 1997;168:333-8.

17. Romei C, Tavanti L, Sbragia P, De Liperi A, Carrozzi L, Aquilini F, et al. Idiopathic interstitial pneumonias: do HRCT criteria established by ATS/ ERS/JRS/ALAT in 2011 predict disease progression and prognosis? Radiol Med 2015;120:930-40.

18. Pellegrino R, Viegi G, Brusasco V, Crapo RO, Burgos F, Casaburi R, et al. Interpretative strategies for lung function tests. Eur Respir J 2005;26: 948-68.

19. ATS statement: guidelines for the six-minute walk test. Am J Respir Crit Care Med 2002;166:111-7.

20. Ishikawa N, Hattori N, Yokoyama A, Tanaka S, Nishino R, Yoshioka K, et al. Usefulness of monitoring the circulating Krebs von den Lungen- 6 levels to predict the clinical outcome of patients with advanced nonsmall cell lung cancer treated with epidermal growth factor receptor tyrosine kinase inhibitors. Int J Cancer 2008;122:2612-20.

21. Matsuno Y, Satoh H, Ishikawa H, Kodama T, Ohtsuka M, Sekizawa K. Simultaneous measurements of KL- 6 and SP-D in patients undergoing thoracic radiotherapy. Med Oncol 2006;23:75-82.

22. Fraser CG, Hyltoft Petersen P, Libeer JC, Ricos C. Proposals for setting generally applicable quality goals solely based on biology. Ann Clin Biochem 1997;34(Pt 1):8-12.

23. Tate J and Ward G. Interferences in immunoassay. Clin Biochem Rev 2004;25:105-20.

24. Sakamoto K, Taniguchi H, Kondoh Y, Johkoh T, Sumikawa H, Kimura T, et al. Serum KL-6 in fibrotic NSIP: correlations with physiologic and radiologic parameters. Respir Med 2010;104:127-33.

25. Zhu C, Zhao YB, Kong LF, Li ZH, Kang J. The expression and clinical role of KL-6 in serum and BALF of patients with different diffuse interstitial lung diseases. Zhonghua Jie He He Hu Xi Za Zhi 2016;39:93-7.

26. Bonella F, Volpe A, Caramaschi P, Nava C, Ferrari P, Schenk K, et al. Surfactant protein $D$ and $\mathrm{KL}-6$ serum levels in systemic sclerosis: correlation with lung and systemic involvement. Sarcoidosis Vasc Diffuse Lung Dis 2011;28:27-33.

27. Kuwana M, Shirai Y, Takeuchi T. E Elevated serum Krebs von den Lungen- 6 in early disease predicts subsequent deterioration of pulmonary function in patients with systemic sclerosis and interstitial lung disease. J Rheumatol 2016;43:1825-31.

28. Raghu G and Selman M. Nintedanib and pirfenidone. New antifibrotic treatments indicated for idiopathic pulmonary fibrosis offer hopes and raises questions. Am J Respir Crit Care Med 2015;191:252-4.

29. Yokoyama A, Kohno N, Hamada H, Sakatani M, Ueda E, Kondo K, et al. Circulating KL-6 predicts the outcome of rapidly progressive idiopathic pulmonary fibrosis. Am J Respir Crit Care Med 1998;158:1680-4. 